Marquette University

e-Publications@Marquette

Biological Sciences Faculty Research and

Publications

Biological Sciences, Department of

$11-1997$

\title{
Effect of 17 Days of Bed Rest on Peak Isometric Force and Unloaded Shortening Velocity of Human Soleus Fibers
}

Jeffrey J. Widrick

Marquette University

Janell Romatowski

Marquette University, janell.romatowski@marquette.edu

James L. W. Bain

Medical College of Wisconsin

Scott W. Trappe

Ball State University

Todd A. Trappe

Ball State University

See next page for additional authors

Follow this and additional works at: https://epublications.marquette.edu/bio_fac

Part of the Biology Commons

\section{Recommended Citation}

Widrick, Jeffrey J.; Romatowski, Janell; Bain, James L. W.; Trappe, Scott W.; Trappe, Todd A.; Thompson, Joyce L.; Costill, David L.; Riley, Danny A.; and Fitts, Robert H., "Effect of 17 Days of Bed Rest on Peak Isometric Force and Unloaded Shortening Velocity of Human Soleus Fibers" (1997). Biological Sciences Faculty Research and Publications. 452.

https://epublications.marquette.edu/bio_fac/452 


\section{Authors}

Jeffrey J. Widrick, Janell Romatowski, James L. W. Bain, Scott W. Trappe, Todd A. Trappe, Joyce L. Thompson, David L. Costill, Danny A. Riley, and Robert H. Fitts 
Marquette University

e-Publications@Marquette

\section{Department of Biology Faculty Research and Publications/College of Arts and Sciences}

This paper is NOT THE PUBLISHED VERSION; but the author's final, peer-reviewed manuscript. The published version may be accessed by following the link in the citation below.

American Journal of Physiology: Cell Physiology, Vol. 273, No. 5 (November, 1997): C1690-C1699. DOI. This article is (C) American Physiological Society and permission has been granted for this version to appear in e-Publications@Marquette. American Physiological Society does not grant permission for this article to be further copied/distributed or hosted elsewhere without the express permission from American Physiological Society.

\section{Effect of 17 Days of Bed Rest on Peak Isometric Force and Unloaded Shortening Velocity of Human Soleus Fibers}

\section{J. J. Widrick}

Department of Biology, Marquette University, Milwaukee

J. G. Romatowski

Department of Biology, Marquette University, Milwaukee

L. W. Bain

Department of Cellular Biology and Anatomy, Medical College of Wisconsin, Milwaukee, Wisconsin

S. W. Trappe

Human Performance Laboratory, Ball State University, Muncie, Indiana

T. A. Trappe

Human Performance Laboratory, Ball State University, Muncie, Indiana

J. L. Thompson

Department of Cellular Biology and Anatomy, Medical College of Wisconsin, Milwaukee, Wisconsin 


\section{L. Costill}

Human Performance Laboratory, Ball State University, Muncie, Indiana

D. A. Riley

Department of Cellular Biology and Anatomy, Medical College of Wisconsin, Milwaukee, Wisconsin

\section{R. H. Fitts}

Department of Biology, Marquette University, Milwaukee, Wisconsin

\section{Abstract}

The purpose of this study was to examine the effect of prolonged bed rest (BR) on the peak isometric force $\left(\mathrm{P}_{\mathrm{o}}\right)$ and unloaded shortening velocity $\left(V_{0}\right)$ of single $\mathrm{Ca}^{2+}$-activated muscle fibers. Soleus muscle biopsies were obtained from eight adult males before and after 17 days of $6^{\circ}$ head-down BR. Chemically permeabilized single fiber segments were mounted between a force transducer and position motor, activated with saturating levels of $\mathrm{Ca}^{2+}$, and subjected to slack length steps. $V_{\mathrm{o}}$ was determined by plotting the time for force redevelopment vs. the slack step distance. Gel electrophoresis revealed that $96 \%$ of the pre- and $87 \%$ of the post-BR fibers studied expressed only the slow type I myosin heavy chain isoform. Fibers with diameter $>100 \mu \mathrm{m}$ made up only $14 \%$ of this post-BR type I population compared with $33 \%$ of the pre-BR type I population. Consequently, the post-BR type I fibers ( $n=147)$ were, on average, $5 \%$ smaller in diameter than the pre-BR type I fibers $(n=218)$ and produced $13 \%$ less absolute $P_{0}$. BR had no overall effect on $P_{o}$ per fiber cross-sectional area $\left(P_{o} / C S A\right)$, even though half of the subjects displayed a decline of $9-12 \%$ in $\mathrm{P}_{\mathrm{o}} / \mathrm{CSA}$ after BR. Type I fiber $V_{\mathrm{o}}$ increased by an average of $34 \%$ with BR. Although the ratio of myosin light chain 3 to myosin light chain 2 also rose with BR, there was no correlation between this ratio and $V_{0}$ for either the pre- or post-BR fibers. In separate fibers obtained from the original biopsies, quantitative electron microscopy revealed a $20-24 \%$ decrease in thin filament density, with no change in thick filament density. These results raise the possibility that alterations in the geometric relationships between thin and thick filaments may be at least partially responsible for the elevated $V_{0}$ of the post-BR type I fibers.

in the rat, the absence of normal weight-bearing activity results in a rapid decline in the mass of the hindlimb extensor muscles. As little as 6 days of spaceflight or 7 days of ground-based hindlimb suspension is sufficient to reduce the soleus mass of this species by $25-35 \%(2,8)$. These atrophied soleus muscles display altered contractile characteristics, including a reduction in peak force per unit muscle mass, an increase in maximal shortening velocity, and a reduction in peak power output $(2,8)$. At least a portion of this change in soleus function is the result of alterations in processes of muscle contraction that lie distal to sarcoplasmic reticulum $\mathrm{Ca}^{2+}$ release $(9,21,22,34)$.

Humans exposed to chronic non-weight bearing may also experience a reduction in lower limb muscular performance. Declines in voluntary peak isometric ankle extensor torque ranging from $~ 15-40 \%$ have been reported following long- and short-term spaceflight and prolonged bed rest $(14,19)$. However, due to the complexity of the intact neuromuscular system, it is often difficult to ascribe these changes in human muscle performance to specific physiological mechanisms. The development of effective interventions to combat reductions in muscle function will require a more complete understanding of how the physiological processes of muscle contraction are altered by chronic non-weight bearing.

In the present study, we used single chemically permeabilized muscle fiber segments, activated with saturating levels of $\mathrm{Ca}^{2+}$, to investigate whether prolonged bed rest altered cross-bridge mechanisms of muscle contraction. We were specifically interested in whether the peak isometric force $\left(P_{o}\right)$ and unloaded shortening velocity $\left(V_{0}\right)$ of single soleus fibers were affected by bed rest, since $P_{o}$ falls and $V_{0}$ rises for rat soleus fibers 
following non-weight bearing $(9,22,34)$. The soleus was selected for study because, in the rat, single fibers from this slow-twitch muscle show the greatest functional responses to the absence of weight bearing activity (9). The duration of bed rest examined in this study, 17 days, corresponded with the duration of the National Aeronautics and Space Administration (NASA) Life and Microgravity Sciences (LMS) Space Shuttle mission (STS78 , June 20 to July 7,1996$)$. This was done to allow future evaluation of bed rest as a ground-based model of spaceflight, since similar single fiber experiments are being conducted on muscle fibers obtained before and after the LMS mission.

\section{METHODS}

\section{Subjects}

This study was approved by the Institutional Review Boards at Marquette University, the Medical College of Wisconsin, Ball State University, and NASA Ames Research Center. Eight adult males were selected from a pool of volunteers to serve as subjects. Each subject underwent a medical examination and provided written informed consent before participation. The mean $( \pm S E)$ age, height, and pre-bed rest body mass of these individuals were $43 \pm 3$ years, $182 \pm 2 \mathrm{~cm}$, and $82.2 \pm 4.3 \mathrm{~kg}$, respectively. For simplicity, we have identified these individuals as subjects 1-8. To facilitate comparison to other studies, the present identification numbers18 correspond to NASA identification numbers $428,439,435,249,405,457,374$, and 420 , respectively.

\section{Bed rest and tissue sampling procedures}

Subjects were continuous residents at the Human Research Facility, NASA Ames Research Center (Moffett Field, CA) for 39 days. Their residence was divided into a 14-day ambulatory control period, a 17-day bed rest period, and a 8-day ambulatory recovery period. Subjects remained at a continuous $6^{\circ}$ head-down tilt throughout the bed rest period. Compliance was monitored $24 \mathrm{~h}$ per day by the staff of the Human Research Facility. All activities, including eating, bathing, excretory functions, and physiological testing, were performed in this position using specially designed facilities and equipment.

One to three days before the start of the control period, a muscle sample was obtained from the left soleus of each subject using the needle biopsy procedure. During the control and bed rest periods, subjects underwent physiological testing using similar equipment and procedures that had been developed for the NASA LMS mission. This testing, which was performed by each subject on two separate days during the control period (days 2 or 3 and days 7 or 8 ) and on three separate occasions during bed rest (days 2 or 3,days 8 or9, and days 13 or 14), included the determination of right ankle extensor isometric torque using a specially designed isokinetic dynamometer and measurement of maximal oxygen consumption during supine cycle ergometry. During each isokinetic testing session, subjects performed 175 voluntary contractions at angular velocities ranging from 0 to $300 \%$, broken down as follows: 12 contractions at peak torque (isometric), 26 contractions at $>50 \%$ but $\leq 80 \%$ of peak torque, $\sim 77$ contractions at $>25 \%$ but $\leq 50 \%$ of peak torque, and $\sim 60$ contractions at $\leq 25 \%$ of peak torque. A complete description of these testing procedures has been presented by Trappe et al. $(32,33)$. A post-bed rest biopsy sample was obtained from the right soleus on the final day of bed rest before reambulation.

Pre- and post-bed rest muscle biopsies were placed on saline-soaked gauze and divided into several portions. One portion, used for the single-fiber functional experiments described in this study, was immediately submerged in cold skinning solution [composition in mM: 125 potassium propionate, 20.0 imidazole ( $\mathrm{pH} 7.0$ ), 2.0 ethylene glycol-bis( $\beta$-aminoethyl ether)- $N, N, N^{\prime}, N^{\prime}$-tetraacetic acid (EGTA), 4.0 ATP, $1.0 \mathrm{MgCl}_{2}$, and $50 \%$ glycerol ( $\mathrm{vol} / \mathrm{vol})]$ and shipped overnight at $4^{\circ} \mathrm{C}$ to Marquette University, where on arrival it was stored at $-20^{\circ} \mathrm{C}$. A second portion was pinned at a mild stretch and immersion fixed in a $0.1 \mathrm{M}$ cacodylate buffer ( $\mathrm{pH} 7.2$ ) consisting of $4 \%$ glutaraldehyde and $2 \%$ paraformaldehyde with $5 \mathrm{mM}$ calcium chloride. This sample was shipped overnight 
at $4^{\circ} \mathrm{C}$ to the Medical College of Wisconsin for osmium postfixation and embedding for electron microscopy as previously described (27).

\section{Single fiber functional experiments}

The compositions of the relaxing and activating solutions were determined with the computer program of Fabiato and Fabiato (4) and the stability constants (adjusted for temperature, $\mathrm{pH}$, and ionic strength) compiled by Godt and Lindley (11). Each solution contained (in mM) 7 EGTA, 20 imidazole, 14.5 creatine phosphate, 1 free $\mathrm{Mg}^{2+}, 4$ free MgATP, and sufficient $\mathrm{KCl}$ and $\mathrm{KOH}$ to produce a total ionic strength of $180 \mathrm{mM}$ and a pH of 7.0. The free $\mathrm{Ca}^{2+}$ concentrations of the relaxing and activating solutions had $\mathrm{pCa}$ values (where $\mathrm{pCa}=-\log$ free $\mathrm{Ca}^{2+}$ concentration) of 9 and 4.5 , respectively.

All contractile measurements were conducted within the initial 28 days after the biopsy procedure. The methods used to mount the single fiber segments were the same as those used previously in this laboratory $(9,22,34,35)$. Briefly, a single fiber segment was isolated from a muscle bundle, transferred to an experimental chamber, and mounted between an isometric force transducer (Cambridge model 400; Cambridge Technology, Watertown, MA) and a direct current position motor (Cambridge model 300B; Cambridge Technology). The experimental apparatus was attached to the stage of an inverted microscope during the experiments. Sarcomere length was adjusted to $2.5 \mu \mathrm{m}$, and the fiber segment length (FL) was recorded. A Polaroid photograph was taken of the fiber while it was briefly suspended in air, and fiber width was measured at three points along the photograph. The mean of these measurements was defined as fiber diameter by assuming the fiber takes on a circular cross section when suspended in air (23). Prior to study, the fiber was briefly bathed in relaxing solution containing 0.5\% Brij-58 (polyoxyethylene 20 cetyl ether; Sigma Chemical, St. Louis, MO).

The fiber was activated by transfer from its original chamber, containing relaxing solution, into an adjacent chamber containing activating solution. These solutions were maintained at $15^{\circ} \mathrm{C}$ throughout data collection. Output from the force transducer and position motor was directed to a digital storage oscilloscope before being amplified and interfaced to a personal computer. Custom software performed on-line analysis and stored data to disks.

Absolute peak force (in $\mathrm{mN}$ ) was calculated as the difference between resting force, measured while the fiber was in relaxing solution, and the maximal force obtained during activation at pCa 4.5. Normalized peak force (in $\mathrm{kN} / \mathrm{m}^{2}$ ) was defined as the absolute peak force divided by the fiber cross-sectional area (CSA). Peak stiffness was determined by oscillating the fiber $(1.5 \mathrm{kHz})$ at an amplitude that produced a $0.05 \%$ peak-to-peak change in $\mathrm{FL}$ ( $\Delta$ length) as previously described (34). Peak elastic modulus (stiffness/fiber CSA) was calculated as [( $\Delta$ force in pCa $4.5-\Delta$ force in pCa 9.0)/( $\Delta$ length) $] \times(F L / f i b e r ~ C S A)$.

$V_{\mathrm{o}}$ was determined by the slack test procedure as previously performed in this laboratory $(9,22,34,35)$. The times required for the redevelopment of force after five to six imposed slack steps (each $\leq 20 \%$ of $F L$ ) were plotted against the corresponding slack length, and the points were fitted with a linear least squares regression line. The slope of this line was $V_{0}$ that was normalized to the length of the fiber and expressed as FL per second.

\section{Single fiber gel electrophoresis}

After the contractile measurements were made, the fiber segment was solubilized in $10 \mu \mathrm{l}$ of $1 \%$ sodium dodecyl

sulfate sample buffer and stored at $-80^{\circ} \mathrm{C}$. To determine fiber myosin heavy chain (MHC) composition, $\sim 0.5 \mathrm{nl}$ of fiber volume was run on a Hoefer SE 600 gel system consisting of a 3\% (wt/vol) acrylamide stacking gel and a 5\% (wt/vol) separating gel (21). To determine myosin light chain (MLC) expression, $\sim 1 \mathrm{nl}$ of fiber volume was loaded on a gel consisting of a $3.5 \%$ acrylamide stacking gel and a $12 \%$ acrylamide separating gel (21). All gels were silver stained as described by Giulian et al. (10). A flatbed scanner with a transparency adapter was used to store 
an image of each gel on computer disk. Image analysis software (SigmaGel, Jandel Scientific Software) was used to quantify the relative levels of $M L C_{1}, M L C_{2}$, and $M L C_{3}$ in each fiber.

\section{Electron microscopy}

Longitudinal and thin cross sections $(\sim 60 \mathrm{~nm})$ were cut from bundles of fibers and stained with uranyl acetate and lead citrate before examination and photographing in a JEOL 100 CXII electron microscope. Myofilament densities and sarcomere length were determined from cross and longitudinal sections, respectively. Final myofilament density values were adjusted for variations in sarcomere lengths on an individual subject basis (1). Interpretations were made on 40 pre- and 40 post-bed rest well-fixed fibers not damaged during dissection (5 pre- and 5 post-bed rest fibers per subject). Processing artifacts in pre-bed rest controls did not generate the ultrastructural changes observed for the post-bed rest tissues in this report.

\section{Data analysis}

Results are presented as mean \pm SE. Pre- and post-bed rest means were compared with analysis of variance. Statistical significance was accepted at $P<0.05$.

\section{RESULTS}

\section{Single fiber MHC expression}

Contractile properties and $\mathrm{MHC}$ composition were determined for a total of 395 single soleus fibers (227 prebed rest, 168 post-bed rest). As illustrated in lane 1 of Fig.1, the $5 \%$ polyacrylamide gel system resolved the slow type I MHC isoform and the two fast MHC isoforms present in adult human skeletal muscle (29). Overall, 218 of the pre-bed rest fibers, or $96 \%$ of the group total, and 147 of the post-bed rest fibers, or $87 \%$ of the group total, expressed type I MHC exclusively. The remaining fibers expressed either a fast MHC isoform (7 pre- and 16 postbed rest fibers with subject 5 accounting for 11 of the fast post-bed rest fibers) or coexpressed slow and fast isoforms ( 2 pre- and 5 post-bed rest fibers). Typically, the fast isoform present in these fibers was type lla, although a small number of post-bed rest fibers expressed the type Ilx isoform. Because the vast majority of the pre- and post-bed rest fiber populations expressed the type I MHC exclusively, the present analysis focuses entirely on the contractile properties of these fibers.

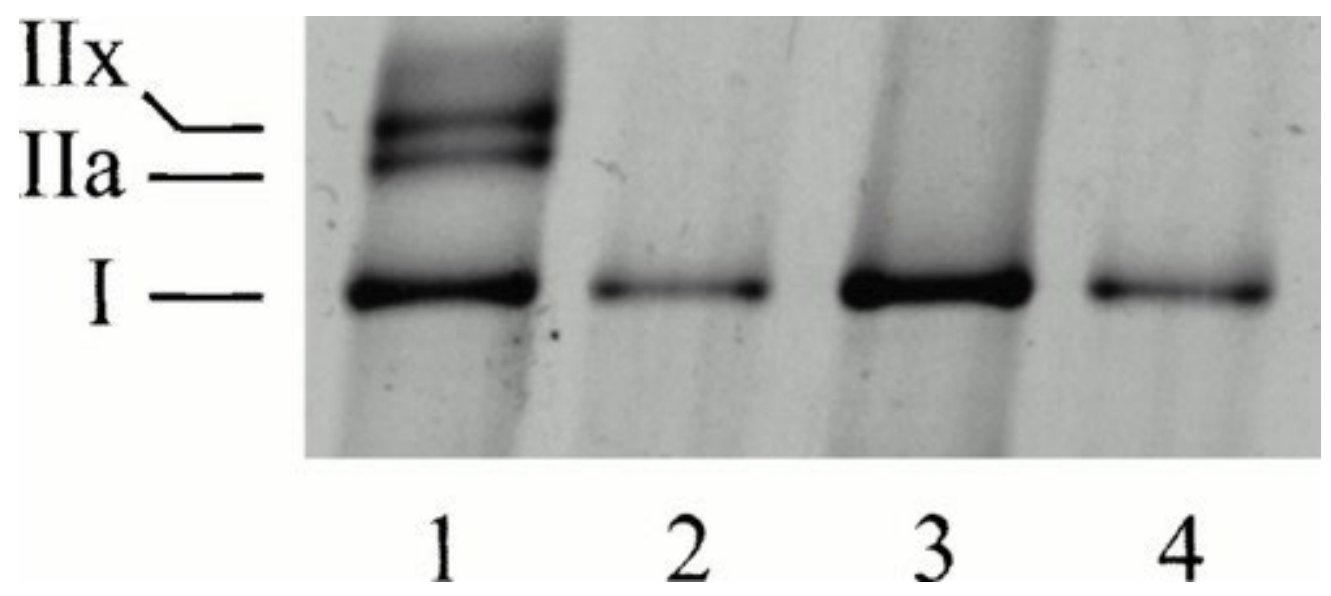

Fig. 1. Representative $5 \%$ polyacrylamide gel illustrating myosin heavy chain (MHC) identification in human single muscle fibers.Lane 1 was loaded with a human skeletal muscle myosin standard. Each of the remaining lanes was loaded with a single human post-bed rest soleus fiber. Values for unloaded shortening velocity $\left(V_{0}\right)$ of fibers in lanes $2-4$ were $1.03,0.72$, and 0.65 fiber segment lengths $(\mathrm{FL}) / \mathrm{s}$, respectively. 


\section{Type I fiber diameter and absolute force}

A positive correlation was observed between peak $\mathrm{Ca}^{2+}$ activated force and fiber diameter for both the pre-bed rest $(r=0.80, P<0.05)$ and post-bed rest $(r=0.61, P<0.05)$ populations (Fig.2). However, on average, the postbed rest fibers were $5 \%$ smaller in diameter $(P<0.05)$ and produced $13 \%$ less peak absolute force $(P<0.05)$ than the pre-bed rest fibers (Table 1 ).



Fig. 2. Relationship between fiber diameter and peak $C a^{2+}$-activated force.A: pre-bed rest type I fibers.B: postbed rest type I fibers. Peak force $(\mathrm{mN})$ was significantly correlated $(P<0.05)$ with fiber diameter for both groups (pre-bed rest, $r=0.80$; post-bed rest, $r=0.61$ ). Number of fibers per group is same as in Table 1.

Table 1. Diameter, peak absolute force, peak normalized force, and unloaded shortening velocity of pre- and post-bed rest type I fibers

Table 1. Diameter, peak absolute force, peak normalized force, and unloaded shortening velocity of pre- and post-bed rest type I fibers

\begin{tabular}{|l|l|l|l|l|l|}
\hline & No. of Fibers & Diameter, $\mu \mathrm{m}$ & $\mathrm{P}_{\mathrm{o}}, \mathrm{mN}$ & $\mathrm{P}_{\mathrm{o}}, \mathrm{kN} / \mathrm{m}^{2}$ & $V_{\mathrm{o}}, \mathrm{FL} / \mathrm{s}$ \\
\hline Pre-bed rest & 218 & $94 \pm 1$ & $0.99 \pm 0.02$ & $139 \pm 2$ & $0.86 \pm 0.02$ \\
\hline Post-bed rest & 147 & $89 \pm 1^{*}$ & $0.86 \pm 0.02^{*}$ & $138 \pm 3$ & $1.15 \pm 0.05^{*}$ \\
\hline
\end{tabular}

Values are means $\pm S E$. $P_{\text {o }}$, peak isometric force; $V_{0}$, unloaded shortening velocity; $F L$, fiber length. 
Significant difference $(P<0.05)$ between pre-bed rest and post-bed rest means.

Figure 2 suggests that the reduction in the mean diameter of the post-bed rest population was due mainly to a decline in the number of large-diameter fibers making up this group. This is confirmed by the histograms in Fig. 3 , in which fibers with diameters $>100 \mu \mathrm{m}$ made up only $14 \%$ of the post-bed rest population in comparison to $33 \%$ of the fiber population before bed rest. There was no indication that bed rest further reduced the diameter of the smallest fibers, as only three post-bed rest fibers, or $2 \%$ of the post-bed rest population, were observed with diameters that were less than the diameter of the smallest of the pre-bed rest fibers. Only five of the eight subjects showed fiber atrophy with bed rest (Fig.4A). However, the slope of the regression line in Fig. $4 A$ was considerably $<1.00$, indicating that those subjects with the largest-diameter pre-bed rest fibers showed the greatest decline in fiber diameter with bed rest.

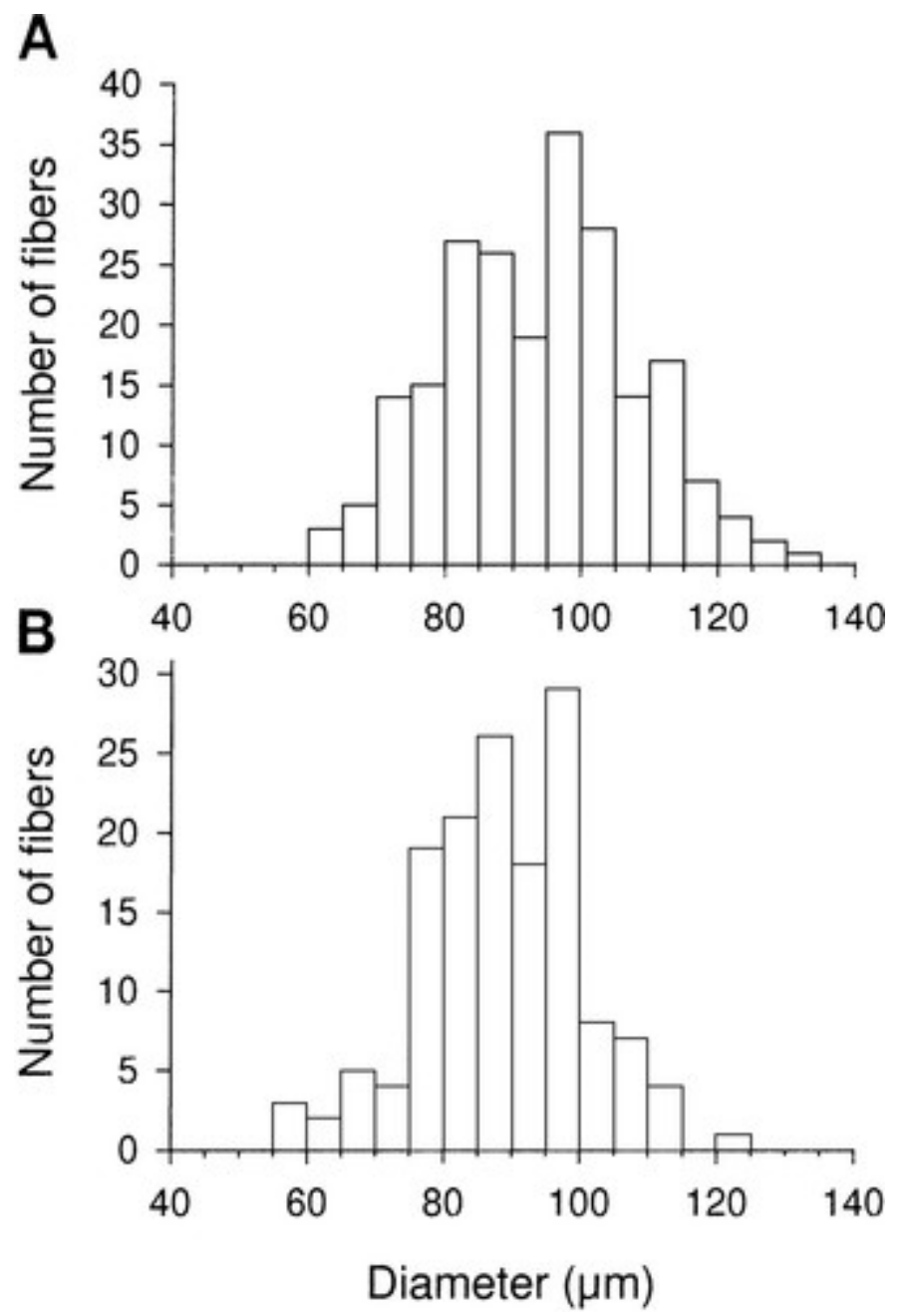

Fig. 3. Frequency distributions of fiber diameter.A: pre-bed rest type I fibers. $B$ : post-bed rest type I fibers. Data were obtained by collapsing the individual data points in Fig. 2 into diameter bins of $5 \mu \mathrm{m}$. Note that fibers with diameters $>100 \mu \mathrm{m}$ made up 33 and $14 \%$ of the pre-bed rest and post-bed rest populations, respectively. 

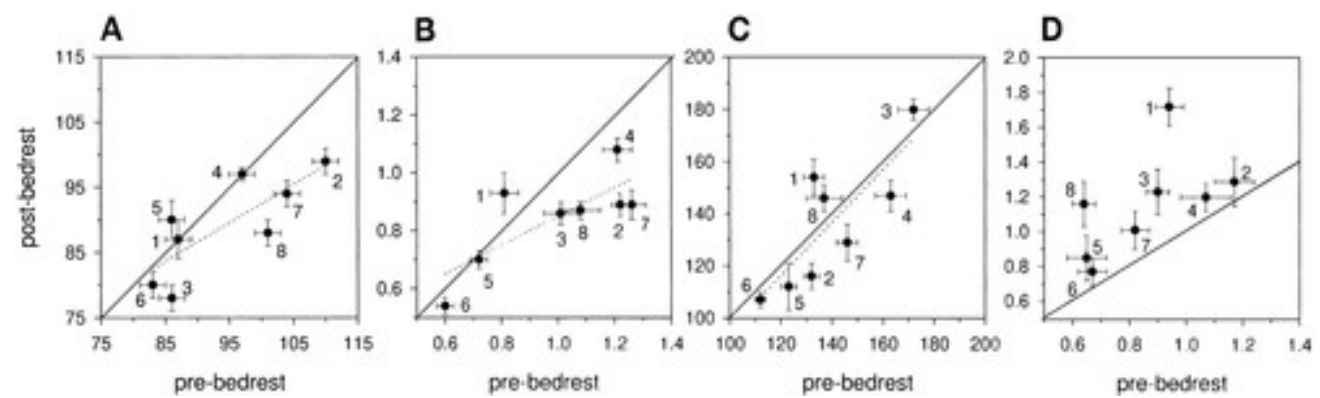

Fig. 4. Relationship between pre-bed rest ( $x$-axes) and post-bed rest ( $y$-axes) type I fiber diameter $(A$, in $\mu \mathrm{m})$, absolute peak isometric force $\left(\mathrm{P}_{\mathrm{o}}, B\right.$, in $\left.\mathrm{mN}\right)$, normalized $\mathrm{P}_{\mathrm{o}}\left(C\right.$, in $\left.\mathrm{kN} / \mathrm{m}^{2}\right)$, and $V_{\mathrm{o}}(D$, in $\mathrm{FL} / \mathrm{s})$ for the individual subjects. Symbols represent means \pm SE values for a particular subject (with the no. adjacent to symbols indicating subject's identification number). Line of identity denoted by solid diagonal line. Significant correlations $(P<0.05)$ were observed between pre- and post-bed rest diameter $(r=0.79)$, absolute $\mathrm{P}_{\mathrm{o}}(r=0.78)$, and normalized $\mathrm{P}_{\mathrm{o}}(r=0.82)$. There was no significant relationship between pre- and post-bed rest $V_{\mathrm{o}}$. Slopes of the least squares regression lines (dotted lines) fit to the pre- and post-bed rest data were $0.59,0.50$, and 1.02 for $A-C$, respectively. Subject sample sizes were as follows (pre-bed rest, post-bed rest, respectively): subject $1=$ 33,13 ; subject $2=31,19$; subject $3=27,21$; subject $4=20,22$; subject $5=27$, 7 ; subject $6=25,19$; subject $7=32$, 24; andsubject $8=23,22$.

Changes in peak absolute force followed a pattern similar to that observed for fiber diameter. Half of the fibers in the pre-bed rest population, but only $27 \%$ of the post-bed rest population, produced peak forces $>1.0 \mathrm{mN}$ (Fig. 5). Fibers from all but one subject produced less average force after bed rest vs. before (Fig. 4B). 


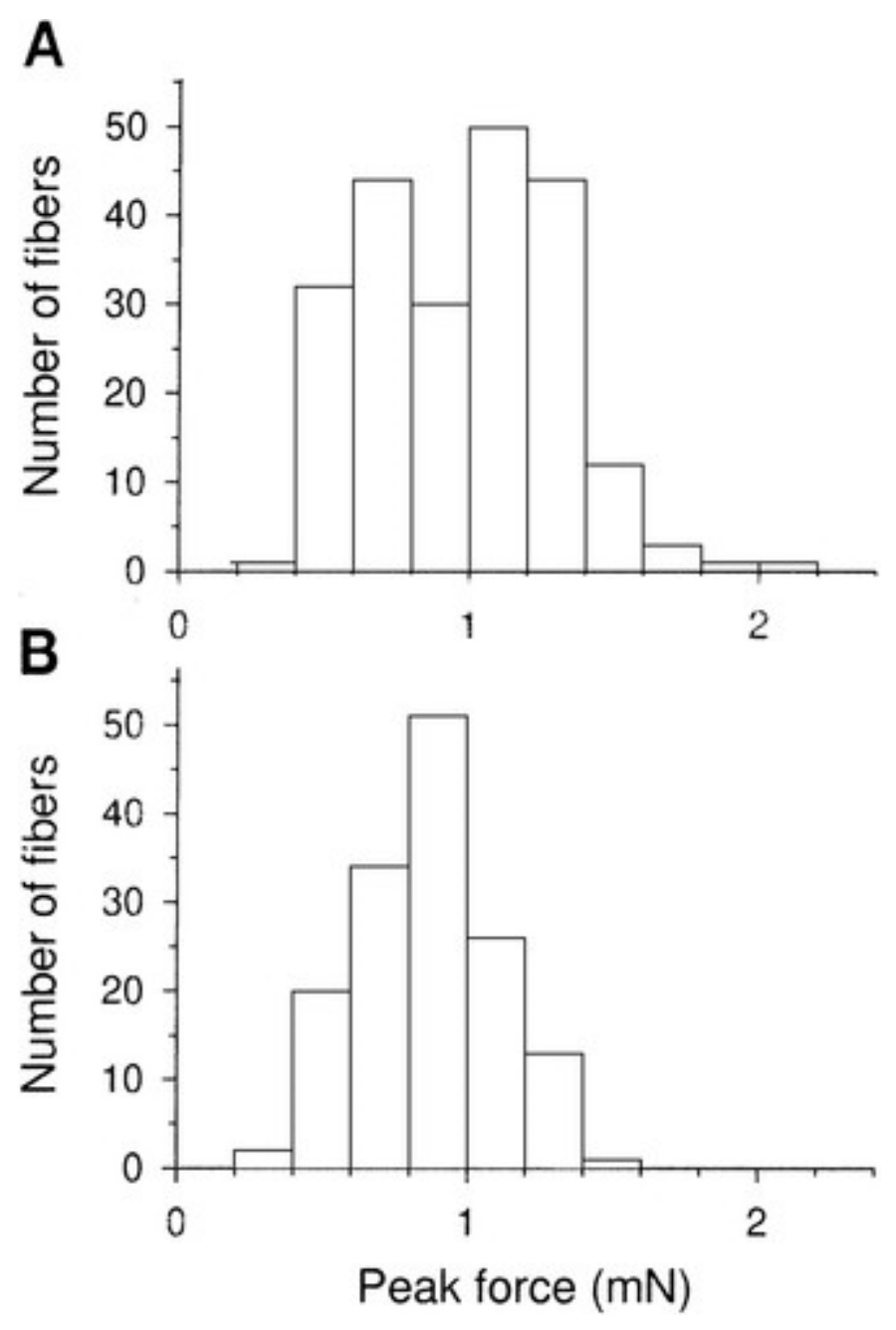

Fig. 5. Frequency distributions of peak $\mathrm{Ca}^{2+}$-activated force.A: pre-bed rest type I fibers.B: post-bed rest type I fibers. Data were obtained by collapsing the individual data points in Fig. 2 into force bins of $0.2 \mathrm{mN}$. Fibers with peak force $>1 \mathrm{mN}$ made up 51 and $27 \%$ of the pre-bed rest and post-bed rest populations, respectively.

\section{Peak normalized fiber force}

On average, peak absolute force declined in proportion to fiber atrophy and there was no change in peak normalized force following bed rest (Table 1). The linear regression line describing the relationship between preand post-bed rest mean values for individual subjects has a slope of 1.02 and almost overlaps the line of identity (Fig.4C). However, four individuals displayed a 9-12\% reduction in normalized force and one a drop of $4 \%$.

\section{Type I fiber peak stiffness}

Stiffness was determined on 104 pre-bed rest and 89 post-bed rest fibers obtained from six of the eight subjects (subjects 1-4,7, and8). Peak elastic modulus decreased by $22 \%$ with bed rest (for pre-bed rest, $2.51 \pm 0.07$ $\mathrm{kN} / \mathrm{m}^{2} \times 10^{4}$; for post-bed rest, $\left.1.95 \pm 0.06 \mathrm{kN} / \mathrm{m}^{2} \times 10^{4} ; P<0.05\right)$. Because no difference in peak normalized force was observed between these 104 pre- and 89 post-bed rest fibers, the ratio of peak isometric tension to elastic modulus increased by $20 \%$ with bed rest (51 \pm 1 for pre-bed rest and $61 \pm 1$ for post-bed rest; $P<0.05$ ).

Type I fiber Vo 
The average $V_{o}$ of the post-bed rest type I soleus fibers was $34 \%$ greater than the pre-bed rest type I mean (Table 1). This overall increase in $V_{0}$ was the result of a group of high-velocity type I fibers in the post-bed rest population that was absent in the pre-bed rest population (Fig.6). A closer examination of Fig. 6 reveals that only one pre-bed rest type I fiber had a $V_{\text {o }}$ that exceeded $1.80 \mathrm{FL} / \mathrm{s}$. In contrast, 25 of the post-bed rest type I fibers, or $17 \%$, had a $V_{0}>1.80 \mathrm{FL} / \mathrm{s}$. Every subject showed an overall increase in $V_{0}$ with bed rest (Fig. $4 D$ ). There was no significant relationship between pre-bed rest and post-bed rest $V_{0}$ for the eight subjects.

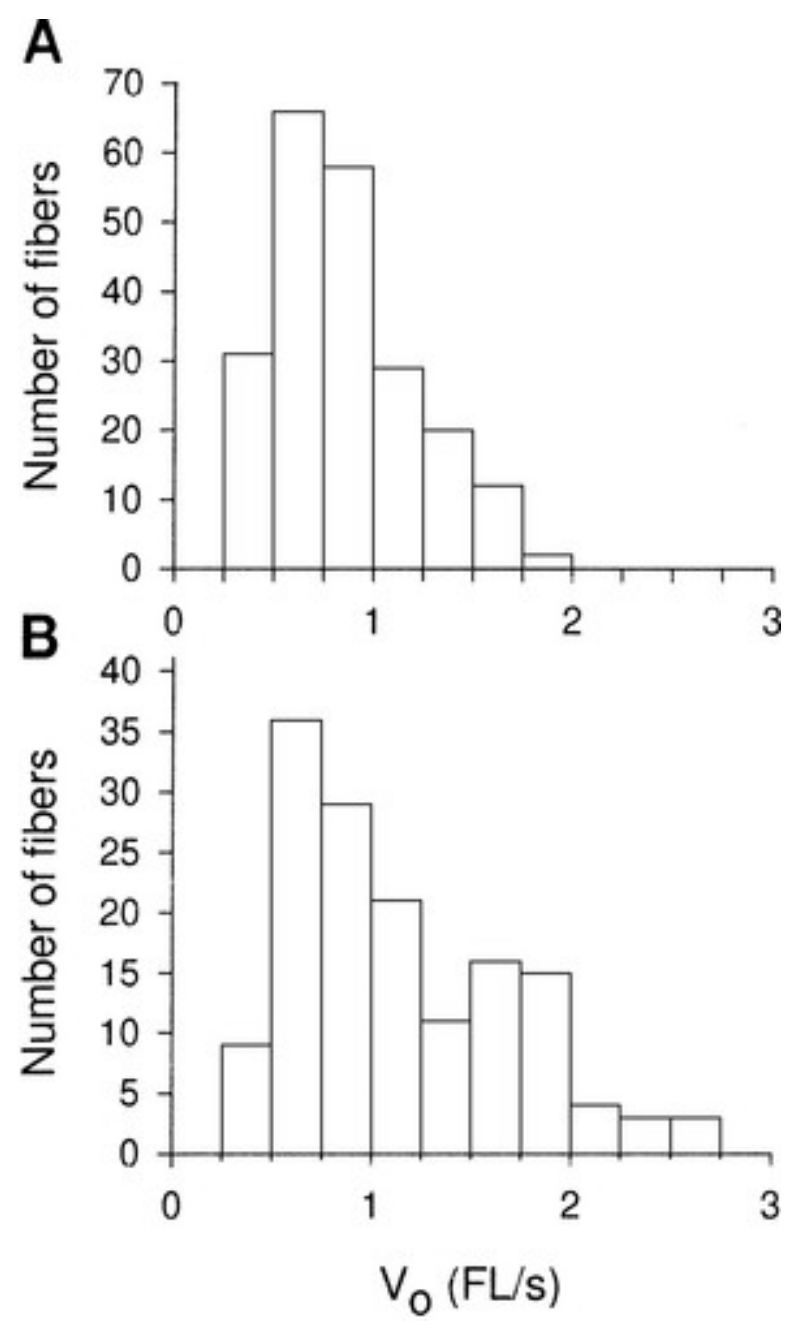

Fig. 6. Frequency distributions of fiber $V_{0} . A$ : pre-bed rest type I fibers. $B$ : post-bed rest type I fibers. Number of fibers for each distribution is same as in Table 1 . Only $1 \%$ of the pre-bed rest fibers had $a V_{0}>1.8 \mathrm{FL} / \mathrm{s}$ compared with $17 \%$ of the post-bed rest fibers.

\section{Fiber MLC composition}

Figure 7 is a representative $12 \%$ gel illustrating the MLC isoform expression of three soleus fibers. In this example, the two soleus fibers expressing type I MHC expressed only slow isoforms of $M^{2} C_{1}$ and MLC $C_{2}$ Table2 is a summary of the MLC composition of 182 pre- and 90 post-bed rest type I fibers, all of which expressed only the slow $\mathrm{MLC}_{1}$ and $\mathrm{MLC}_{2}$ isoforms. Note that the average $V_{0}$ values of the pre- and post-bed rest fibers making up this subset were representative of the entire pre- and post-bed rest fiber population. 


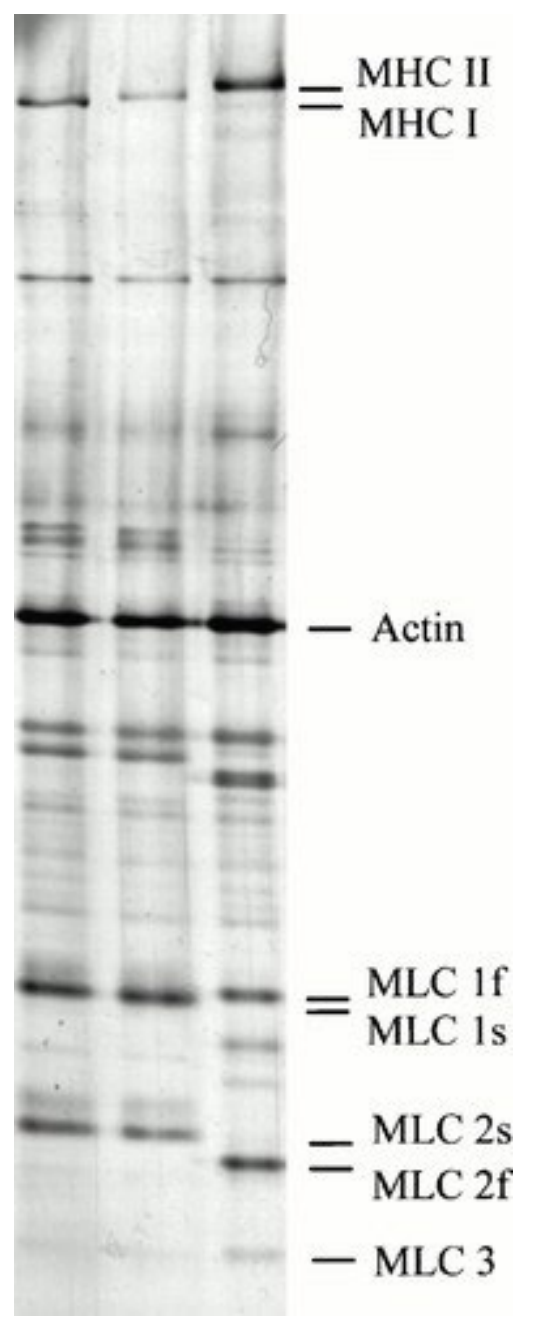

Fig. 7. Representative $12 \%$ gel illustrating myosin light chain (MLC) expression in human single muscle fibers. Single fiber inright lane expressed type II MHC and fast isoforms of $\mathrm{MLC}_{1}$ and $\mathrm{MLC}_{2}$. Two remaining fibers expressed type I MHC and slow isoforms of $\mathrm{MLC}_{1}$ and $\mathrm{MLC}_{2}$. Note lower level of $\mathrm{MLC}_{3}$ expression in type I fibers.

Table 2. Myosin light chain isoform composition of pre-bed rest and post-bed rest type I fibers

\begin{tabular}{|l|l|l|l|l|l|l|}
\hline Condition & No. of Fibers & $\mathrm{MLC}_{1 \mathrm{~s}}$ & $\mathrm{MLC}_{2 \mathrm{~s}}$ & $\mathrm{MLC}_{3}$ & $\mathrm{MLC}_{3} / \mathrm{MLC}_{25}$ & $\mathrm{~V}_{0}, \mathrm{FL} / \mathrm{s}$ \\
\hline Pre-bed rest & 182 & $0.525 \pm 0.004$ & $0.411 \pm 0.004$ & $0.064 \pm 0.003$ & $0.159 \pm 0.009$ & $0.87 \pm 0.03$ \\
\hline Post-bed rest & 90 & $0.515 \pm 0.004$ & $0.407 \pm 0.004$ & $0.078 \pm 0.004$ & $0.198 \pm 0.012^{*}$ & $1.22 \pm 0.06^{*}$ \\
\hline
\end{tabular}

Values are means \pm SE. Myosin light chain (MLC) isoform content was expressed relative to the total MLC of the fiber.

${ }^{*}$ Significant difference $(P<0.05)$ between pre-bed rest and post-bed rest means.

There was no difference in the relative expression of $\mathrm{MLC}_{1 \mathrm{~s}}, \mathrm{MLC}_{2 \mathrm{~s}}$, or $\mathrm{MLC}_{3}$ between pre- and post-bed rest fibers. Because each myosin S-1 expresses one regulatory $M L C\left(M L C_{2}\right)$ and one essential MLC (either $M L C_{1}$ or $\mathrm{MLC}_{3}$ ), we examined possible changes in the essential MLC composition of these single fibers by calculating the ratio of $\mathrm{MLC}_{3}$ to $\mathrm{MLC}_{2 \mathrm{~s}}(17)$. This ratio was elevated $25 \%$ in the post-bed rest population. However, there was no relationship between fiber $V_{\circ}$ and the $\mathrm{MLC}_{3}$-to-MLC ${ }_{25}$ ratio either before or after bed rest (Fig.8). 


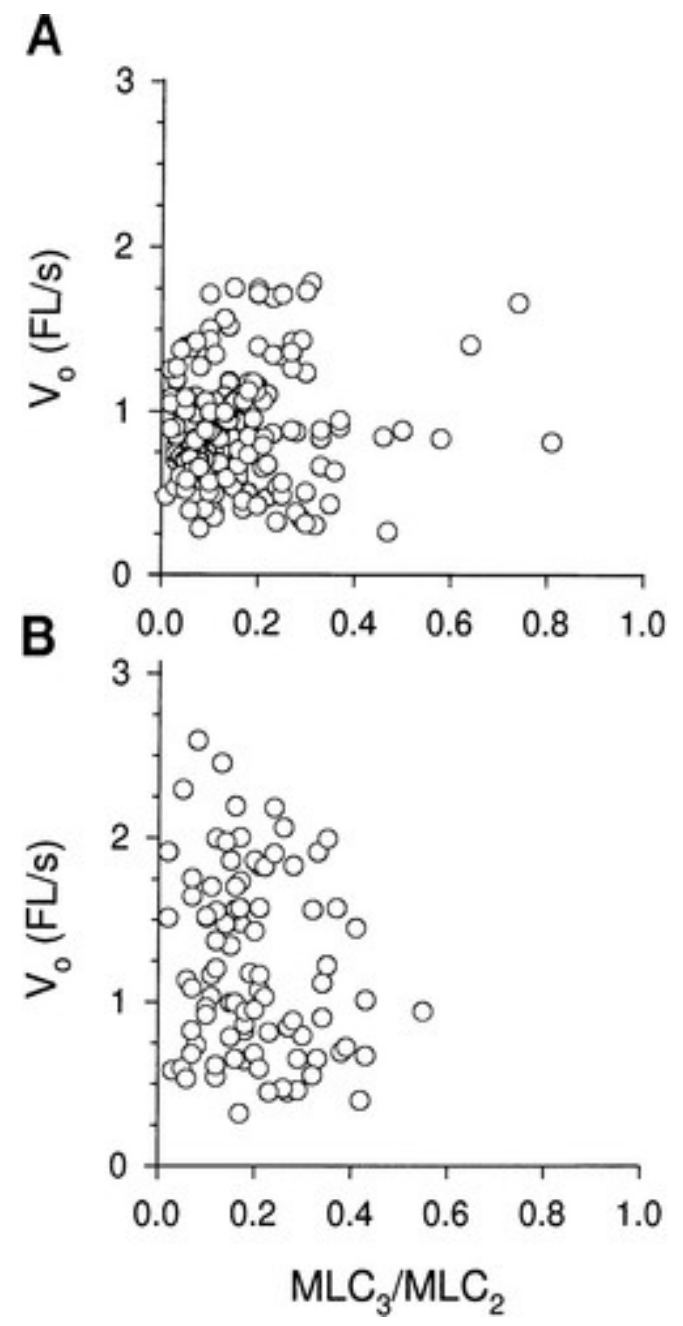

Fig. 8. Relationship between $V_{\circ}$ and the $\mathrm{MLC}_{3}$ content of type I fibers.A: pre-bed rest fibers.B: post-bed rest fibers. $\mathrm{MLC}_{3}$ expression is expressed relative to the regulatory light chain content $\left(\mathrm{MLC}_{25}\right)$. No significant relationship $(P>$ 0.05 ) was observed between $V_{0}$ and $\mathrm{MLC}_{3}$ expression for both the pre-bed rest and post-bed rest populations. Mean values for these data are compiled in Table 2.

\section{Myofibrillar ultrastructure}

Electron micrographs of longitudinally sectioned pre- and post-bed rest soleus fibers are presented in Fig. 9. After bed rest, the A bands of soleus myofibrils exhibited normal density, whereas the I bands appeared "motheaten." This morphological alteration indicates that actin filaments were eliminated to a greater extent than myosin filaments with bed rest. An extensive ultrastructural quantitation of the thick and thin filament concentrations demonstrated no average change in thick filament density with bed rest but a $20 \%$ reduction in thin filament density in the I band and a $24 \%$ reduction in thin filament density in the A band (Table3). 


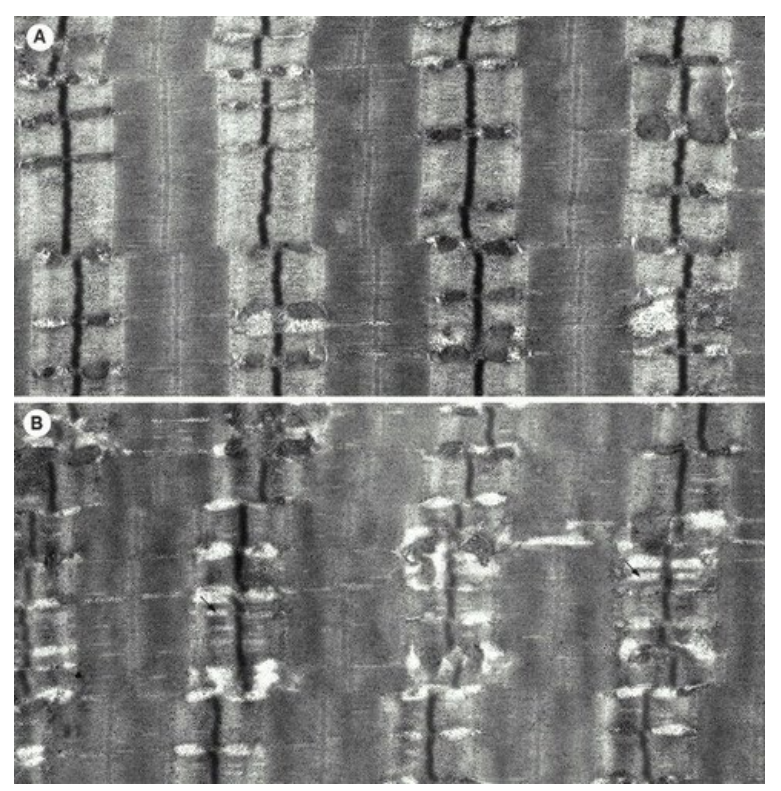

Fig. 9. Electron micrographs of longitudinal sections of pre-bed rest $(A)$ and post-bed rest $(B)$ soleus fibers. There is a decreased myofibril diameter in the post-bed rest fibers as indicated by the shorter $Z$ lines. Sarcomeres in the postbed rest fiber $(B)$ are missing myofilaments in the I band regions (arrows), giving the fiber a moth-eaten appearance. Magnification: $\times 17,750$.

Table 3. Myofilament density of pre-bed rest and post-bed rest fibers

\begin{tabular}{|l|l|l|l|}
\hline Condition & Filaments $/ \mu \mathrm{m}^{2}$ & & \\
\hline & Thin filaments, I band & Thin filaments, A band & Thick filaments \\
\hline Pre & $3,027 \pm 130$ & $2,559 \pm 60$ & $987 \pm 24$ \\
\hline Post & $2,426 \pm 118^{3-150}$ & $1,950 \pm 673-150$ & $993 \pm 39$ \\
\hline
\end{tabular}

Values are means \pm SE of 40 pre- and 40 post-bed rest fibers ( 5 pre- and 5 post-bed rest fibers per subject). Sarcomere length was $2.54 \pm 0.15$ and $2.63 \pm 0.13 \mu \mathrm{m}$ for pre- and post-bed rest fibers, respectively. Filament density values were normalized for variation in sarcomeric length as discussed in METHODS.

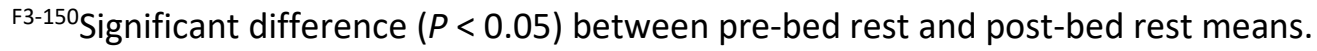

\section{DISCUSSION}

\section{Effect of bed rest on fiber diameter and peak isometric force}

After 17 days of bed rest, the average diameter of the type I fibers obtained from the soleus (measured at a consistent sarcomere length of $2.5 \mu \mathrm{m}$ ) was reduced by $5 \%$. This change, which is equivalent to a $10 \%$ decline in fiber CSA, is almost identical to the $9 \%$ reduction in average fiber CSA obtained from the morphological analysis of sectioned muscle biopsy samples from these same eight subjects (32).

Interestingly, we observed a bed rest-induced reduction in the percentage of large-diameter soleus fibers, i.e., those with diameters $>100 \mu \mathrm{m}$. Because membrane permeabilization is associated with a $20 \%$ increase in fiber diameter (12), these large fibers would have in vivo diameters of $\sim 83 \mu \mathrm{m}$ or greater. Related to this, we found that subjects with the largest-diameter pre-bed rest fibers displayed the greatest degree of post-bed rest fiber atrophy. Similarly, Edgerton et al. (3) noted that the greatest fiber atrophy after an 11-day spaceflight was experienced by those astronauts with the largest-diameter preflight fibers.

Our results suggest that the largest pre-bed rest type I fibers either were more susceptible to non-weight bearing and underwent the greatest atrophy or were more likely to express fast $\mathrm{MHC}$ isoforms after bed rest and, therefore, eliminated from our post-bed rest analysis. The fact that the percentage of type I fibers fell from 
96 to $87 \%$ with bed rest would lend support to this second mechanism. However, $67 \%$ of the post-bed rest type II or I/II fibers were obtained from a single individual (subject 5). If these 12 post-bed rest fibers are eliminated from analysis, type I fibers then account for $95 \%$ of the post-bed rest population, a value virtually identical to the pre-bed rest result. The possibility that our post-bed rest type I fiber distributions were biased by this one individual is supported by a morphological analysis of $>3,000$ sectioned and histochemically stained fibers from these same subjects in which no pre- to post-bed rest change in fiber type distribution was observed (32). Furthermore, the three individuals who had the largest pre-bed rest fibers and displayed the most fiber atrophy (subjects 2,7, and8) accounted for a combined total of one pre-bed rest and two post-bed rest type II or type I/II fibers. These observations do not support the idea of a selective conversion of the largest type I pre-bed rest fibers to type II or I/II post-bed rest fibers but rather that the largest type I fibers were indeed more susceptible to atrophy.

Because peak Ca2+-activated absolute force was directly correlated with fiber diameter before and after bed rest, the more pronounced atrophy of the largest fibers reduced the percentage of fibers producing relatively high force $(>1.0 \mathrm{mN})$, with essentially no change in the proportion of fibers producing relatively low force. Overall, the average decline in peak force production was proportional to the loss of fiber CSA, although one-half of the subjects displayed reductions in normalized force of $\sim 10 \%$.

\section{Effect of bed rest on fiber Vo}

On the basis of $5 \%$ polyacrylamide gel electrophoresis, all of the pre- and post-bed rest fibers making up this study expressed the adult type I MHC exclusively. The average $V_{\circ}$ of these fibers rose by $34 \%$ with bed rest. This increase is quantitatively similar to the change in $V_{0}$ noted for rat type I soleus fibers following 14-21 days of hindlimb suspension $(9,22,34)$.

The average $V_{o}$ of the pre-bed rest fibers, $0.86 \mathrm{FL} / \mathrm{s}$, was greater than the value of $0.52 \mathrm{FL} / \mathrm{s}$ we previously reported for 61 human type I soleus fibers (35). However, an examination of individual mean values indicates that several subjects were in quite good agreement with our previous work (35) as well as our preliminary results from the astronauts of the LMS mission (Widrick and Fitts, unpublished observations). In contrast, some subjects (e.g.,subjects 2 and4) displayed values that were considerably greater than expected. Although we have no clear explanation for this wide range in pre-bed rest $V_{0}$, it should be noted that the eight subjects were extremely heterogeneous in terms of their occupational and recreational activity levels and their pre-bed rest fitness levels. These factors could have contributed to the wide variability in $V_{o}$ observed in this study. Despite greater than expected variation in pre-bed rest type I fiber $V_{0}$, all subjects displayed an increase in this variable after bed rest. Furthermore, there was no significant relationship between a subject's pre-bed rest $V_{\circ}$ and the extent to which this variable rose with bed rest (Fig.4D).

$V_{0}$ is thought to be limited by the rate of actomyosin cross-bridge detachment (16), a process that has been shown to vary with $\operatorname{MHC}(17,26)$ and $\operatorname{MLC}(15,30)$ isoform expression and the physical properties of the myofilament lattice (23). However, it is not clear which of these processes (or combination of processes) is responsible for the elevated post-bed rest $V_{\circ}$ observed in this study.

In the laboratory rat, non-weight bearing is associated with an increase in the number of soleus fibers coexpressing type I and II MHCs (21). Therefore, one possibility is that our gel conditions failed to identify small amounts of fast MHC coexpressed by the post-bed rest fibers. However, Reiser et al. $(25,26)$ have concluded that $V_{\mathrm{o}}$ is a nonlinear function of the relative proportions of slow and fast $\mathrm{MHC}$ isoforms present in a fiber, since slower-cycling cross bridges act as an internal drag on the rate of detachment of faster cycling cross bridges and thereby negate some of the effects of faster-cycling cross bridges. Recent studies conducted on human type Ila, $\mathrm{Ila/IIb}$, and IIb fibers $(17,18)$ and on rhesus monkey type I, I/Ila, and Ila fibers (R. H. Fitts, S. C. Bodine, J. G. Romatowski, and J. J. Widrick, unpublished observations) suggest that $V_{\mathrm{o}}$ remains relatively unchanged until the 
expression of a faster $\mathrm{MHC}$ isoform reaches $20-30 \%$, or more, of total MHC. Although it is possible that our electrophoretic conditions failed to detect $\leq 2.5 \%$ of the total MHC present in a particular fiber (22), this appears to be an order of magnitude below that required to increase $V_{\circ}$ by $34 \%$.

A second possibility is that bed rest modified MLC composition, since alterations in regulatory and essential MLCs are known to have modulatory effects on fiber $V_{0}(15,30)$. This mechanism is not supported by the results of the present study. First, all of the type I pre- and post-bed rest fibers expressed similar levels of the slow $\mathrm{MLC}_{2}$ isoform. Second, although $\mathrm{MLC}_{3}$ content increased following bed rest, levels of this essential light chain were unrelated to $V_{0}$ for both the pre- or the post-bed rest type I fibers. In this regard, our pre-bed rest results are in agreement with the work of Larsson and Moss (17), who also reported no relationship between $\mathrm{MLC}_{3}$ levels and $V_{\mathrm{o}}$ in human slow-twitch muscle fibers.

Another possibility is that bed rest induced de novo or reexpression of an alternative slow MHC isoform. Such an alternative $\mathrm{MHC}$ isoform could be responsible for the elevated $V_{\mathrm{o}}$ of the post-bed rest fibers if this isoform had a greater intrinsic $V_{0}$ than the adult $\beta-\mathrm{MHC}$ and comigrated with the adult $\beta-\mathrm{MHC}$ on our gels. However, even when we employed gel conditions previously shown to resolve two slow $\mathrm{MHC}$ isoforms in young adult rat whole soleus muscles (5), we observed only one slow $\mathrm{MHC}$ isoform. Thus it seems unlikely that the increased post-bed rest $V_{\text {o }}$ was induced by the expression of a second slow MHC previously observed by Fauteck and Kandarian (5).

A final possibility pertains to the relationship between myofilament geometry and fiber shortening velocity. Riley et al. (27) have reported that, after hindlimb suspension, myofibrils of rat soleus fibers exhibit a motheaten appearance, suggesting a reduction in myofilament packing. Because it is known that fiber $V_{\mathrm{o}}$ is extremely sensitive to changes in myofilament lattice spacing (23), we quantified the density of thick and thin filaments in a separate group of histologically fixed pre- and post-bed rest fibers obtained from the original muscle biopsies. Whereas thick filament density remained constant, thin filament density declined by $20-24 \%$ in the post-bed rest fibers. In an idealized myofilament, this would be approximated by the loss of one thin filament for every six thin filaments, i.e., a single thick filament would be surrounded by five thin filaments instead of the normal six. Because the area occupied by these five filaments remains constant (no change in myosin density), plane geometry dictates that the average distance from thin to thick filaments increases.

One limitation of this approach is that we do not have myofilament density measurements and physiological data on the same fibers. However, the observed increase in $V_{0}$, the decline in elastic modulus, and the rise in the ratio of $\mathrm{P}_{\mathrm{o}}$ to elastic modulus are all consistent with an increase in the distance between thin and thick filaments $(13,23)$. Furthermore, a relatively wide change in $V_{o}$ coupled with a much smaller change in $P_{o}$ is similar to results obtained from single fiber preparations in which filament lattice spacing is experimentally altered (23).

\section{Comparison to previous human studies}

To our knowledge, this is the first study to investigate the effects of bed rest on the functional properties of human soleus fibers. Previously, Larsson et al. (18) studied human vastus lateralis fibers obtained after 42 days of bed rest and found a reduction in peak normalized force with no change in type I fiber $V_{0}$. Comparisons between these two studies must take into account differences in experimental design, particularly the muscles studied and the duration of bed rest. For instance, the soleus and the vastus lateralis are under different anatomic constraints during the bed rest model of non-weight bearing. The subjects in the present study were observed to frequently extend and maintain the foot at a $40^{\circ}$ plantar flexed position during bed rest (24). Consequently, the soleus was maintained at a shorter than normal length for much of the bed rest period. In contrast, the vastus lateralis is either at its normal weight-bearing length or stretched (if subjects flex the knee) during bed rest. It is well established that muscles maintained in neutral or lengthened positions do not display the same changes in protein metabolism (20), ultrastructure (27), or contractile function (28) observed for muscles maintained at short lengths. Single fibers obtained from muscles that are chronically shortened may 
therefore display different contractile characteristics than fibers from muscles maintained at neutral or lengthened positions.

In the rat hindlimb suspension model, rates of protein degradation and synthesis follow distinct time courses (31). It seems likely that human skeletal muscle protein metabolism may also vary with the duration of bed rest. The fact that we observed no reduction in normalized peak force whereas Larsson et al. (18) observed a $40 \%$ reduction in this variable could be the result of an increase in net protein degradation as bed rest is extended beyond 17 days. Interestingly, one-half of our subjects showed a bed rest-induced reduction in normalized force of $9-12 \%$, suggesting that, in many individuals, the loss in contractile protein had begun to exceed fiber atrophy by the 17 th day of non-weight bearing.

Finally, to mimic the LMS space shuttle mission, the present subjects performed loaded contractile activity during the isokinetic and aerobic capacity testing sessions. On the basis of the observations of Edgerton et al. (3), it seems unlikely that the aerobic capacity test affected our results. The five sessions of isokinetic testing conducted between the pre- and post-bed rest biopsies averaged out to only 28 contractions per day.

Furthermore, only 4 of these 28 contractions were performed above $50 \%$ of peak (voluntary) isometric force and of these 4 only 2 were performed as maximal isometric contractions. Clearly, this was insufficient activity to prevent the atrophy and functional changes noted in the single fibers of this study. However, we have no way of knowing whether the observed responses would have been exacerbated if this isokinetic testing activity had been absent.

\section{Comparison to previous animal studies}

The most widely studied model of non-weight bearing is ground-based rat hindlimb suspension, with the soleus being the most frequently studied muscle. The $34 \%$ increase in post-bed rest type I soleus fiber $V_{0}$ in the present study is very similar to the $30-50 \%$ increase reported for rat type I soleus fiber after 14 days of hindlimb suspension $(9,22,34)$. However, the decline in the absolute force of Ca2+-activated rat type I fibers $(22,34)$ is four times greater than the absolute force deficits noted for the human fibers in the present study. In addition, rat type I soleus fibers show a significant reduction in peak normalized force after non-weight bearing $(9,22,34)$. The greater force decline in rat fibers may be the result of interspecies differences in the net rates of protein degradation during non-weight bearing $(6,31)$.

\section{Individual responses to bed rest}

Subject 1 appeared to react to bed rest in a manner that was inconsistent with the responses of the other seven subjects. Subject 1 displayed no atrophy and the largest increases in both normalized $P_{\circ}$ and $V_{\circ}$ with bed rest. This individual was also the only subject to show an increase in absolute $\mathrm{P}_{\mathrm{o}}$ with non-weight bearing. It is unclear why this individual responded in this way. Regardless of the mechanism involved, elimination ofsubject 1 had no effect on a reanalysis of the fiber diameter, $P_{0}$, and $V_{0}$ data of the remaining seven individuals [for 185 pre- and 134 post-bed rest type I fibers, diameters were $96 \pm 1$ and $90 \pm 1 \mu \mathrm{m}(P<0.05)$, absolute $P_{\circ}$ values were $1.02 \pm$ 0.02 and $0.85 \pm 0.02 \mathrm{mN}(P<0.05)$, normalized $P_{\circ}$ values were $140 \pm 2$ and $137 \pm 3 \mathrm{kN} / \mathrm{m}^{2}(P>0.05)$, and $V_{\circ}$ values were $0.85 \pm 0.03$ and $1.10 \pm 0.05(P<0.05)$, respectively].

\section{Summary and conclusions}

Seventeen days of bed rest produced an average decline in type I fiber diameter of $5 \%$ and a reduction in peak $\mathrm{Ca}^{2+-a c t i v a t e d}$ force of $13 \%$, with relatively large-diameter, and therefore high-force-producing, fibers being most susceptible to atrophy. On average, fiber atrophy was proportional to reductions in $\mathrm{Ca}^{2+}$-activated force so that peak force per fiber CSA was unchanged. After bed rest, $V_{\circ}$ and $\mathrm{MLC}_{3}$ content were elevated 34 and $25 \%$, respectively, in single fibers expressing type I MHC. However, $\mathrm{MLC}_{3}$ content was unrelated to $V_{0}$ in these slowtwitch fibers. The mechanism underlying the elevated post-bed rest $V_{\mathrm{o}}$ is unclear. Possibilities include the 
expression of an alternative type I MHC or changes in the geometric properties of the myofilament lattice. Support for the second of these mechanisms comes from our finding of a $20-24 \%$ reduction in thin filament density following bed rest.

This study was supported by NASA Grant NAS9-18768 (to R. H. Fitts). D. A. Riley received partial salary support from NASA Grant NAG2-956.

\section{AUTHOR NOTES}

Address for reprint requests: R. H. Fitts, Marquette Univ., Dept. of Biology, Wehr Life Sciences Bldg., PO Box 1881, Milwaukee, WI 53201-1881.

\section{REFERENCES}

1 Brandt P. W., Lopez E., Reuben J. P., Grundfest H.The relationship between myofilament packing density and sarcomere length in frog striated muscle.J. Cell Biol.331967255263

2 Caiozzo V. J., Baker M. J., Herrick R. E., Tao M., Baldwin K. M.Effect of spaceflight on skeletal muscle: mechanical properties and myosin isoform content of a slow muscle.J. Appl. Physiol.76199417641773

3 Edgerton V. R., Zhou M.-Y., Ohira Y., Klitgaard H., Jiang B., Bell G., Harris B., Saltin B., Gollnick P. D., Roy R. R., Day M. K., Greenisen M.Human fiber size and enzymatic properties after 5 and 11 days of spaceflight.J. Appl. Physiol. 78199517331739

4 Fabiato A., Fabiato F.Calculator programs for computing the composition of the solutions containing multiple metals and ligands used for experiments in skinned muscle cells.J. Physiol. Paris 751979463505

5 Fauteck S. P., Kandarian S. C.Sensitive detection of myosin heavy chain composition in skeletal muscle under different loading conditions.Am. J. Physiol.268Cell Physiol. 371995C419C424

6 Ferrando A. A., Lane H. W., Stuart C. A., Davis-Street J., Wolfe R. R.Prolonged bed rest decreases skeletal muscle and whole body protein synthesis.Am. J. Physiol.270Endocrinol. Metab. 331996E627E633

8 Fitts R. H., Metzger J. M., Riley D. A., Unsworth B. R.Models of disuse: a comparison of hindlimb suspension and immobilization.J. Appl. Physiol.60198619461953

9 Gardetto P. R., Schluter J. M., Fitts R. H.Contractile function of single muscle fibers after hindlimb suspension.J. Appl. Physiol.66198927392749

10 Giulian G. G., Moss R. L., Greaser M.Improved methodology for analysis and quantitation of proteins on onedimensional silver-stained slab gels.Anal. Biochem.1291983277287

11 Godt R. E., Lindley B. D.Influence of temperature upon contractile activation and isometric force production in mechanically skinned muscle fibers of the frog.J. Gen. Physiol.801982279297

12 Godt R. E., Maughan D. W.Influence of osmotic compression on calcium activation and tension in skinned muscle fibers of the rabbit.Pflügers Arch.3911981334337

13 Goldman Y. E., Simmons R. M.The stiffness of frog skinned muscle fibres at altered lateral filament spacing.J. Physiol. (Lond.)3781986175194

14 Grigoryeva L. S., Kozlovskaya I. B.Effect of weightlessness and hypokinesia on velocity and strength properties of human muscles.Kosm. Biol. Aviakosm. Med.2119872730

15 Hofmann P. A., Metzger J. M., Greaser M. L., Moss R. L.Effects of partial extraction of light chain 2 on the $\mathrm{Ca}^{2+}$ sensitivities of isometric tension, stiffness, and velocity of shortening in skinned skeletal muscle fibers.J. Gen. Physiol.951990477498

16 Huxley A. F.Muscle structure and theories of contraction.Prog. Biophys. Biophys. Chem. 71957255318 
17 Larsson L., Moss R. L.Maximum velocity of shortening in relation to myosin isoform composition in single fibres from human skeletal muscles.J. Physiol. (Lond.) 4721993595614

18 Larsson L., Xiaopeng L., Berg H. E., Frontera W. R.Effects of removal of weight-bearing function on contractility and myosin isoform composition in single human skeletal muscle cells.Pflügers Arch.4321996320328

19 LeBlanc A., Gogia P., Schneider V., Krebs J., Schonfeld E., Evans H.Calf muscle area and strength changes after five weeks of horizontal bed rest.Am. J. Sports Med.161988624629

20 Loughna P., Goldspink G., Goldspink D. F.Effect of inactivity and passive stretch on protein turnover in phasic and postural rat muscles.J. Appl. Physiol.611986173179

21 McDonald K. S., Blaser C. A., Fitts R. H.Force-velocity and power characteristics of rat soleus muscle fibers after hindlimb suspension.J. Appl. Physiol.77199416091616

22 McDonald K. S., Fitts R. H.Effect of hindlimb unweighting on single soleus fiber maximal shortening velocity and ATPase activity.J. Appl. Physiol.74199329492957

23 Metzger J. M., Moss R. L.Shortening velocity in skinned single muscle fibers. Influence of filament lattice spacing.Biophys. J.521987127131

24 Narici M., Kayser B., Barattini P., Cerretelli P.The effect of 17 day head-down bedrest on human triceps surae contractile properties (Abstract).J. Physiol. (Lond.)499.P199781P

25 Reiser P. J., Kasper C. E., Greaser M. L., Moss R. L.Functional significance of myosin transitions in single fibers of developing soleus muscle.Am. J. Physiol.254Cell Physiol. 231988C605C613

26 Reiser P. J., Moss R. L., Giulian G. G., Greaser M. L.Shortening velocity in single fibers from adult rabbit soleus muscles is correlated with myosin heavy chain composition.J. Biol. Chem.260198590779080

27 Riley D. A., Slocum G. R., Bain J. L. W., Sedlak F. R., Sowa T. E., Mellender J. W.Rat hindlimb unloading: soleus histochemistry, ultrastructure, and electromyography.J. Appl. Physiol.6919905866

28 Simard C. P., Spector S. A., Edgerton V. R.Contractile properties of rat hind limb muscles immobilized at different lengths.Exp. Neurol.771982467482

29 Smerdu V., Karsch-Mizrachi I., Campione M., Leinwand L., Schiaffino S.Type Ilx myosin heavy chain transcripts are expressed in type Ilb fibers of human skeletal muscle.Am. J. Physiol.267Cell Physiol. 361994C1723C1728

30 Sweeney H. L., Kushmerick M. J., Mabuchi K., Sréter F. A., Gergely J.Myosin alkali light chain and heavy chain variations correlate with altered shortening velocity of isolated skeletal muscle fibers.J. Biol.

Chem.263198890349039

31 Thomason D. B., Biggs R. B., Booth F. W.Protein metabolism and $\beta$-myosin heavy-chain mRNA in unweighted soleus muscle.Am. J. Physiol.257Regulatory Integrative Comp. Physiol. 261989R300R305

32 Trappe S. W., Trappe T. A., Costill D. L., Fitts R. H.Human calf muscle function in response to 17-days of bed rest (Abstract).Med. Sci. Sports Exerc.281996S146

33 Trappe T. A., Trappe S. W., Costill D. L., Fitts R. H.Time course of cardiovascular deconditioning with 17 days of $6^{\circ}$ head down tilt bed rest (Abstract).Med. Sci. Sports Exerc.281996S145

34 Widrick J. J., Bangart J. J., Karhanek M., Fitts R. H.Soleus fiber force and maximal shortening velocity after non-weight bearing with intermittent activity.J. Appl. Physiol.801996981987

35 Widrick J. J., Romatowski J. G., Karhanek M., Fitts R. H.Contractile properties of rat, rhesus monkey, and human type I muscle fibers.Am. J. Physiol.272Regulatory Integrative Comp. Physiol. 411997R34R42 